\title{
Atuação da enfermagem no autocuidado e reabilitação de pacientes Que sofreram Acidente Vascular Encefálico
}

\author{
Nursing activities in self-care and rehabilitation of patients who suffered Stroke
}

Actuación de la enfermería en el autocuidado y rehabilitación de pacientes que sufreran Accidente Vascular Encefalico

\author{
Juliana Cristina Lessmann', Fernanda De Conto", Greice Ramos"', \\ Míriam Susskind Borenstein', Betina Homer Schlindwein Meirelles ${ }^{1}$
}

'Universidade Federal de Santa Catarina. Programa de Pós-graduação em Enfermagem. Florianópolis, SC "Universidade Federal de Santa Catarina. Hospital Universitário Ernani Polydoro de São Tiago. Florianópolis, SC

IIIPrefeitura Municipal de Florianópolis. Programa de Saúde da Família. Florianópolis, SC

Submissão: 20/09/2009

Aprovação: 05/09/2010

\section{RESUMO}

Quando o cérebro sofre alterações vasculares podem ocorrer sérias seQuelas motoras, sensoriais e na percepção. Nesta perspectiva o estudo objetiva relatar/ compartilhar assistência de Enfermagem ao indivíduo acometido pelo Acidente Vascular Encefálico enfatizando a reabilitação e o autocuidado baseado em Orem. Trata-se de relato de experiência de prática assistencial, realizada de maio a julho de 2006, com 15 indivíduos e seus familiares, em um centro de reabilitação da região sul do Brasil. O cuidado de Enfermagem integral ocorre com atividades de estimulação sensitiva, motora, controle postural, amplitude de movimentos e treino de marcha, sempre acompanhados por ações de educação em saúde. Estas habilidades conferem aos enfermeiros capacidades de atuação proporcionando a redução de danos e incapacidades.

Descritores: Enfermagem; Acidente cerebrovascular; Autocuidado.

\section{ABSTRACT}

There can be serious damage to motor, sensatory, and perceptive capabilities when the brain suffers vascular alterations. With this in mind, the objective of this study is to report and share Nursing care to the individual who has suffered a cerebral vascular accident, emphasizing Orem-based rehabilitation and self-care. This study is a report of a practical care experiment, carried out in May and July of 2006 involving 15 individuals and their families in a rehabilitation center in southern Brazil. Integral Nursing care occurs with stimulation to sensatory, motor, postural control, movement amplitude, and marching practice activities when accompanied by health care education actions. These abilities grant nurses performance capacities, in turn proportioning reductions in damage and incapacities.

Key words: Nursing; Stroke; Self Care.

\section{RESUMEN}

Cuando o cerebro sufre alteraciones vasculares pueden ocurrir serias secuelas motoras, sensoriales y en la percepción. En esta perspectiva el estudio objetiva relatar/compartir asistencia de Enfermería al individuo afectado por accidente vascular cerebral enfatizando la rehabilitación y autocuidado basado en Orem. Se trata de relato de experiencia de práctica asistencial, realizada de mayo a julio de 2006, con 15 individuos y sus familiares, en un centro de rehabilitación de la región sur do Brasil. El cuidado de Enfermería integral ocurre con actividades de estimulación sensitiva, motora, control postural, amplitud de movimientos y entrenamiento de marcha, siempre acompañados por acciones de educación en salud. Estas habilidades confieren a los enfermeros capacidades de actuación, participando en la reducción de daños e incapacidades.

Descriptores: Enfermería; Accidente cerebrovascular; Autocuidado.

AUTOR Juliana Cristina Lessmann. Rua João Pio Duarte Silva 94, apto 502, CEP 88037-000. Florianópolis, SC. CORRESPONDENTE E-mail: julianalessmann@gmail.com 


\section{INTRODUÇÃO}

O cérebro é um órgão extremamente complexo Que desempenha o controle e modulação das funções corpóreas, além de ser o locus da atividade cognitiva e intelectual do ser humano. Composto por inúmeras ligações entre neurônios, Que se comunicam por meio de impulsos elétricos e Químicos, o cérebro pode reconhecer diversos estímulos, sendo capaz de analisá-los e decodificá-los, elaborando respostas às variadas situações ${ }^{(1)}$, sendo fundamental à vida e à capacidade de sobrevivência do ser humano(2).

Entretanto, pode ser acometido por sérias alterações, como um Acidente Vascular Encefálico (AVE), Que caracteriza-se pela diminuição total ou parcial de fluxo sanguíneo em determinada área do cérebro ${ }^{(1,3)}$. O AVE constitui a terceira causa de morte no mundo ${ }^{(4-5)}$, ocorrendo com maior incidência no sexo masculino, em indivíduos com descendência afroamericana e idade superior a 65 anos $^{(1)}$.

Os déficits neurológicos decorrentes do AVE variam conforme a localização da lesão vascular, do tempo de perfusão inadeQuada e da existência de circulação colateral (1). Assim, estes eventos podem acarretar em perda de força, sensibilidade, capacidade de movimentação e controle de diversas áreas corporais, além de acarretar em distúrbios de linguagem, perda do equilíbrio ou coordenação, distúrbios visuais, bem como a perda do controle dos esfíncteres anal e vesical (1). Tais sequelas freeuentemente comprometem a autoestima e autoimagem do indivíduo, bem como sua interação com a família e a sociedade (2).

As seQuelas motoras do AVE apresentam três fases: a fase flácida, a espástica e a de recuperação. Na fase flácida ocorre a perda ou diminuição de força e movimento muscular por paralisia e/ ou fraqueza, sendo que a musculatura tem sua capacidade de contração afetada, resultando na produção de força insuficiente para a realização de movimentos voluntários ${ }^{(6)}$.

A fase espástica é marcada pela transição da flacidez para um estado hiperreflexão muscular e de tendões(7), sendo Que as características de músculos espásticos incluem a resistência ao alongamento e o tônus mais alto do que o normal. Nesta fase é necessário tratamento multiprofissional visando à reeducação muscular, a fim de evitar problemas secundários como disfunções articulares e dolorosas ${ }^{(6)}$

A fase de recuperação, ocorrida por meio da reconstituição de neuro-mecanismos Que executam as funções cerebrais afetadas pelo AVC, é alcançada apenas por meio de intervenções terapêuticas Que auxiliam o indivíduo a restabelecer funções corporais afetadas ${ }^{(6)}$.

Na maioria dos casos, os indivíduos com AVE são hospitalizados para a realização de investigações acerca da origem, gravidade e grau de comprometimento das funções corporais, para realizarem o tratamento e prevenção de seQuelas. Há estudos Que afirmam Que o adeQuado atendimento de saúde, bem como o início precoce de assistência em saúde e reabilitação, podem melhorar consideravelmente o prognóstico da doença ${ }^{(1,3,8)}$.

A reabilitação é uma das inúmeras funções da enfermagem, Que busca a independência para a realização do autocuidado, sendo o segundo entendido como um conjunto de ações desenvolvidas pelo indivíduo e pela família para atender as necessidades da vida diária, Que é aprendido e aperfeiçoado ao longo da vida ${ }^{(9)}$. A reabilitação neurológica em enfermagem é um processo dinâmico, envolvendo orientações para a saúde Que auxiliam os indivíduos doentes e/ou com incapacidades a obterem uma melhor recuperação em todos os sentidos: físico, mental, espiritual e social. Isto possibilita uma melhor Qualidade de vida, Que inclui a recuperação da dignidade, do auto-respeito e independência( ${ }^{(1)}$.

O presente tema constitui relevância à enfermagem contemporânea por abordar a reabilitação de uma doença Que tem grande impacto na saúde do indivíduo, bem como em seu relacionamento familiar e social. Diante do exposto, este texto tem como objetivo relatar/compartilhar a experiência de cuidado de enfermagem desenvolvido junto ao indivíduo acometido pelo AVC, com ênfase na reabilitação e autocuidado tendo por base a Teoria Geral de Enfermagem de Orem.

\section{MARCO TEÓRICO}

A Teoria Geral de Enfermagem de Orem é formada por três construtos teóricos inter-relacionados: a teoria do autocuidado, a teoria do déficit de autocuidado e a teoria dos sistemas de enfermagem ${ }^{(9)}$

Na Teoria do Autocuidado é abordada a prática de cuidados realizados pelo indivíduo com a finalidade de manutenção da condição vital e de saúde ${ }^{(9)}$. O autocuidado é reconhecido como uma habilidade humana, sendo Que sua execução está diretamente ligada às condições Que cada indivíduo possui para autocuidar-se. São apontadas três categorias de exigências terapêuticas/requisitos para o autocuidado: universais, desenvolvimentais e de desvio de saúde ${ }^{(9)}$.

A Teoria de Déficit de Autocuidado, determina Que a enfermagem é necessária Quando um indivíduo é incapaz ou tem limitações na provisão de autocuidado efetivo ${ }^{(10)}$. Nestas situações, o indivíduo necessita adQuirir conhecimentos e habilidades a fim de incorporálos em seu sistema de cuidado.

O último construto da Teoria Geral de Enfermagem de Orem é a Teoria de Sistemas de Enfermagem, Que estabelece a estrutura e orienta as práticas de enfermagem ${ }^{(9)}$. Nela são identificados: o sistema totalmente compensatório; o sistema parcialmente compensatório e o sistema de apoio-educação( ${ }^{(9)}$. Esses sistemas são compostos por elementos fundamentais como: a dimensão da responsabilidade da Enfermagem nas situações de atendimento à saúde; o papel geral e específico de cada participante visando suprir as exigências terapêuticas ${ }^{(9)}$.

\section{MÉTODO}

Tratou-se de um relato de experiência acerca de prática assistencial desenvolvida por três acadêmicas, durante a VIII Unidade Curricular do Curso de Graduação em Enfermagem da Universidade Federal de Santa Catarina, Que resultou no trabalho de conclusão de curso intitulado "O cuidado de enfermagem e o processo de reabilitação do indivíduo acometido pelo Acidente Vascular Encefálico, sob a ótica da Teoria Geral de Enfermagem de Orem"(11).

Desenvolvida de maio a julho de 2006, em um centro de reabilitação vinculado ao Sistema Único de Saúde (SUS), sendo referência para o estado de Santa Catarina.

A assistência ocorreu no período matutino, com no mínimo dois e no máximo Quatro momentos de assistência de enfermagem 
integral, enfocando a reabilitação, bem como a elaboração do Processo de Enfermagem segundo a Teoria de Orem.

Os sujeitos do estudo foram 15 indivíduos acometidos pelo AVE e seus familiares, sendo oito homens e sete mulheres, com idades entre 47 e 75 anos, com tempo transcorrido de 45 dias a 5 anos do último AVE. É importante ressaltar Que todos tinham diagnóstico médico de hipertensão arterial sistêmica e $60 \%$ destes possuíam Diabetes Mellitus. A maioria apresentava comprometimento motor por estar na fase espástica, bem como dificuldade para a realização das atividades da vida diária, dependência de outras pessoas para o cuidado e alteração nos órgão do sentido, principalmente o tato. Um destes também possuía perda de capacidade de expressão verbal e dificuldade de controle dos esfíncteres anal e vesical.

A atuação das acadêmicas de enfermagem cumpriu integralmente os critérios estabelecidos pela Resolução $n^{\circ}$ 196/96, do Conselho Nacional de Saúde ${ }^{(12)}$, mantendo respeito aos aspectos éticos, sendo Que os envolvidos ou seus responsáveis foram convidados a participar e assinaram o Termo de Consentimento Livre e Esclarecido.

\section{APRESENTAÇÃO DAS ATIVIDADES REALIZADAS E DISCUSSÃO}

Quando pensamos em reabilitação como forma de aQuisição de habilidades antigas, significa muito mais do Que reaprender a andar e vestir-se, ou transferir-se da cama para a cadeira de rodas. As atividades Que precisam ser reaprendidas abrangem principalmente redescobrir eual o novo papel do indivíduo dentro da família, para poder enfrentar a realidade e os novos problemas do cotidiano. Esses fatores tornam a reabilitação um processo único e específico para cada ser humano e a enfermagem procura atender estas demandas nos aspectos físicos, psicológicos e psicossociais.

O enfoeue do cuidado também está voltado para a família, sendo necessário Que estes aprendam novos métodos conforme suas demandas, sendo que os ensinamentos necessitam ser contínuos e objetivos, promovendo o reconhecimento da importância das ações, a compreensão do processo e do despertar da participação e colaboração de todos em prol do mesmo objetivo.

As atividades de reabilitação efetuadas pela enfermagem aos indivíduos acometidos pelo AVE são amparadas pela Lei do Exercício Profissional $n^{\circ} 7.498$, de 25 de junho de 1986, por meio do artigo oitavo, inciso I. Sendo que o Código de ética dos profissionais de Enfermagem, aprovado pela Resolução do Conselho Federal de Enfermagem n²40/00 ampara, no capítulo III artigo I 8, a busca e exercício de conhecimentos Que beneficiem a clientela atendida. Fatos Que reforçam a importância da compreensão das atividades de reabilitação desempenhadas por Enfermeiros.

A seguir, apresentamos os principais cuidados realizados, considerando o fortalecimento das aptidões para o autocuidado e as ações de enfermagem na reabilitação em indivíduos com comprometimento neurológico decorrente de AVE.

\section{O cuidado de enfermagem e o controle postural.}

As alterações do controle muscular, da sensibilidade corpórea ou da própriocepção, decorrentes do AVE, podem gerar a perda ou redução da força e movimento muscular, caracterizando a fase flácida ${ }^{(6)}$. Frente a esta etapa, foi realizado posicionamento no leito, mudança de decúbito e alongamento de membros efetuados de forma passiva ou ativa por meio de educação em saúde ${ }^{(5)}$. Para isto primava-se pelo posicionamento anatômico utilizando instrumentos como coxins, almofadas, travesseiros e órteses.

Estes cuidados fazem-se necessários, pois a manutenção de postura inadequada gera alterações que podem causar dor, além de disfunções musculares e articulares $^{(6)}$ Que podem levar o indivíduo a vivenciar a fase mais severa das sequelas motoras do AVC, definida como fase espástica.

\section{O cuidado de enfermagem e os exercícios de amplitude do movimento}

Indivíduos com sequelas decorrentes de AVE, geralmente apresentam debilidades de movimentação causadas pela redução da amplitude articular e da musculatura ${ }^{(6)}$. Assim foi possível proporcionar a manutenção da amplitude de movimentos aplicando e ensinando atividades de alongamento, de forma passiva, Quando as autoras realizaram estes exercícios nos indivíduos acometidos pelo AVE, ou de forma ativa Quando os próprios indivíduos efetuaram os exercícios ${ }^{(5)}$.

Foi realizada orientação a todos os indivíduos, bem como seus familiares, acerca da realização de alongamentos com a finalidade de manutenção da amplitude de movimentação, principalmente em membros inferiores e superiores.

\section{$O$ cuidado de enfermagem no treino de marcha}

Alterações nos padrões de marcha são frequentes em indivíduos com algum tipo de sequela motora nos membros inferiores. Podem ocorrer alterações no perfil e ritmo do ciclo de passadas oriundas da perda ou diminuição do controle da musculatura ${ }^{(6)}$.

A enfermagem pode utilizar-se do sistema de apoio-educação de Orem $^{(9)}$ para realizar ações de educação em saúde acerca da forma com Que os indivíduos deveriam conduzir a marcha. Assim, inicialmente os indivíduos que efetuaram o treino de equilíbrio seguravam-se em barras, ou em outro suporte estável. Quando a capacidade de equilíbrio aumentava, estes aprenderam a alternar o peso corporal entre os membros inferiores ${ }^{(7)}$. Assim Que conseguiram efetuar esta transferência de peso iniciou-se o processo de caminhada em barras paralelas.

Faz-se necessário Que os profissionais de enfermagem, juntamente com a equipe multidisciplinar, efetuem orientações acerca da prevenção de Quedas em caso de treino de marcha realizado no domicílio.

No momento em Que os indivíduos encontravam-se aptos para caminhar foi recomendado o uso de andador e posteriormente da bengala de Quatro pontos ${ }^{(7)}$. Nestes casos recomendou-se Que a marcha fosse treinada em ambientes Que possuíssem símbolos estampados no chão como, por exemplo, passadas, Quadrados e linhas retas para Que este indivíduo realizasse o exercício cerebral concomitante ao treino da marcha, sendo possível o uso de esteiras ergométricas, utilizadas com a velocidade diminuída para a realização destes exercícios.

As autoras acompanharam as atividades de treino de marcha utilizando todos estes instrumentos, realizaram orientações e ações de educação em saúde ${ }^{(15)}$ acerca do seu uso seguro no domicílio e da necessidade de treino frequente para o fortalecimento da musculatura dos membros inferiores.

O treino de marcha torna-se completo Quando há a capacidade 
de subir e descer escadas e rampas. Nestas situações, a equipe multiprofissional atuava incentivando Que na subida, a marcha fosse iniciada com o membro mais fortalecido e na descida, com o membro menos fortalecido ${ }^{(7)}$. Estas condutas favorecem a transferência de peso entre os membros, melhorando o equilíbrio, evitando acidentes e Quedas. As autoras acompanharam os treinos de marcha utilizando escadas e rampas junto aos indivíduos acometidos pelo AVC Que eram capazes de realizá-lo.

\section{O cuidado de enfermagem e os exercícios de manutenção de equilíbrio}

Em alguns indivíduos acometidos pelo AVE podem ocorrer distúrbios no equilíbrio, associados à flacidez e/ou espasticidade, bem como ao comprometimento cerebral ${ }^{(7)}$. As autoras efetuaram exercícios de manutenção de equilíbrio utilizando um "tablado móvel”, instrumento com apoio arredondado e Que não se fixa completamente ao chão, movendo-se suavemente. Os indivíduos com maior controle dos membros inferiores foram convidados a posicionar-se sobre esta prancha e, com auxílio mantiveram-se em posição ortostática. Este exercício foi realizado em frente ao espelho para Que os indivíduos visualizassem seu corpo.

\section{O cuidado de enfermagem e a terapia ocupacional}

A terapia ocupacional visa promover a recuperação de lesões neurológicas, pois estimula o cérebro a restabelecer sua capacidade de realização das atividades da vida diária. Para isto os terapeutas ocupacionais ensinam técnicas para execução de tarefas antigas e usam equipamentos para estimular uso do membro afetado, como as órteses e pesos utilizados nos punhos e tornozelos para reduzir a ataxia de braços e pernas ${ }^{(16)}$.

A enfermagem pode inserir-se neste cuidado reforçando os ensinamentos, adaptando-os a realidade do indivíduo no domicílio e estimulando a realização correta e contínua dos mesmos. Durante a prática assistencial foram efetuados diversos atendimentos envolvendo terapia ocupacional, sendo Que as autoras estimularam a sensibilidade superficial e profunda, usando materiais pontiagudos e de diferentes texturas, estimulação visual e perceptiva, usando jogos coloridos. Também estimularam os indivíduo e as famílias para a continuidade destas atividades no domicílio. Estas ações possibilitam a gradual execução das atividades como vestir-se, arrumar-se, lavar-se, cuidar da aparência, alimentar-se e também proporcionam a melhoria das relações sociais ${ }^{(5)}$.

\section{Enfermagem enfocando a reabilitação e o uso da Teoria de Orem}

A reabilitação é uma das inúmeras funções da enfermagem, Que busca no indivíduo a independência para a realização do autocuidado. A habilidade para realizá-lo é frequentemente a chave para a independência, para o retorno ao lar e para a vida comunitária. Assim Quanto mais precoce é iniciado o processo de reabilitação, melhores são as possibilidades de recuperação do indivíduo ${ }^{(1)}$. Aliado a estes conhecimentos, a aplicação da Teoria Geral de Enfermagem de Orem conferiu maior Qualidade e cientificidade ao cuidado, visto Que os indivíduos com AVE apresentam em sua maioria, limitações para exercer atividades do cotidiano e de reabilitação.

\section{CONSIDERAÇÕES FINAIS}

A realização desta prática assistencial corrobora Que a participação da enfermagem nas atividades de reabilitação, com a promoção da capacidade de autocuidado e melhoria da Qualidade de vida das pessoas com AVE. Também percebe-se a importância da atuação da enfermagem junto à equipe multiprofissional, promovendo a interdisciplinaridade e a troca de saberes Que culmina na realização de assistência integral e de Qualidade às pessoas ${ }^{(17)}$.

Com esta prática assistencial foi possível compreender a importância das atividades de reabilitação e da conscientização das famílias para tais cuidados. Quando o indivíduo reconhece suas potencialidades, sendo estimulado para as ações de autocuidado, torna-se mais ativo e participativo, refletindo positivamente na autoimagem e auto-estima.

O referencial teórico de Orem serviu de instrumento para o reconhecimento dos déficits de autocuidado, viabilizando a elaboração de planejamento das atividades de enfermagem e para a provisão de tais cuidados, além de ter fornecido apoio para a implementação de atividades de reabilitação.

A compreensão da patogenicidade e das peculiaridades do AVE, assim como suas características e fatores determinantes, conferem aos profissionais de saúde, incluindo os Enfermeiros, habilidades para atuar junto ao indivíduo acometido, proporcionando maiores oportunidades de redução dos danos e incapacidades, promovendo uma melhor Qualidade de vida bem como um viver melhor e mais feliz.

\section{REFERÊNCIAS}

I. Smeltzer SC, Bare BG, Brunner LS, Suddarth DS. Tratado de Enfermagem médico-cirúrgica. $10^{\mathrm{a}}$ ed. Rio de laneiro: Guanabara Koogan; 2005.

2. Zarco LA, González F, Oral Casas I. Tratamiento actual del ataque cerebrovascular isquémico (ACV) agudo. Univ Méd Bogotá 2008; 49(4): 467-98.

3. André C. Manual de AVC. Rio de Janeiro: Revinter; 1999.

4. Cordova RAM, Cesarino CB, Tognola WA. Avaliação clínica evolutiva de pacientes pós primeiro Acidente Vascular Encefálico e seus cuidadores. ArQ Ciênc Saúde 2007; 14(2):75-9.

5. Perlini NMOG, Faro ACM. Cuidar da pessoa incapacitada por acidente vascular cerebral no domicílio: o fazer do cuidador familiar. Rev Esc Enfrem USP 2005; 39(2): I 54-63.

6. Ryerson SD. Hemiplegia. In: Umphred DA, organizador. Reabilitação neurológica. $4^{\mathrm{a}}$ ed. Barueri: Manole; 2004. p.782830 .

7. Anderson TP. Reabilitação de pacientes com derrame cerebral completo. In: Kottke FI, Lehmann JF. Tratado de medicina física e reabilitação de Krusen. $4^{\text {a }}$ ed. São Paulo: Manole; 1994. p. 649-99.

8. Costa F, Oliveira S, Magalhães P, Costa B, Papini R, Silveira M, Lang M. Nível de conhecimento da população adulta sobre acidente vascular cerebral (AVC) em Pelotas. I Bras Neurocir 2008; 19(1): $31-7$.

9. Orem DE. Nursing concepts of pratice. $5^{\text {th }}$ ed. Saint Louis: Mosby; 1995. 
10. Foster PC, Bennett AM. Dorothea E. Orem. In: George JB. Teoria de Enfermagem: os fundamentos para a prática profissional. $4^{\mathrm{a}}$ ed. Porto Alegre: Artes Médicas Sul; 2000. p. 83-101.

11. Conto FD, Ramos G, Lessmann IC. O cuidado de Enfermagem e o processo de reabilitação do indivíduo acometido pelo Acidente Vascular Encefálico, sob a ótica da Teoria Geral de Enfermagem de Orem [monografia]. Florianópolis: Curso de Graduação em Enfermagem, Universidade Federal de Santa Catarina; 2006.

12. Ministério da Saúde (BR). Conselho Nacional de Saúde, Comissão Nacional de Ética em Pesquisa. Resolução No 196 de 10 de outubro de 1996: diretrizes e normas regulamentadoras de pesquisas envolvendo seres humanos. Brasília: Ministério da Saúde; 1996.

13. Brasil. Lei No 7.498, de 25 de junho de 1986. Dispões sobre a regulamentação do exercício da Enfermagem e dá providências. Diário Oficial da República Federativa do Brasil 1986 jun 26; 1.

14. Conselho Regional de Enfermagem de Santa Catarina. Legislação sobre o exercício da Enfermagem. Florianópolis: COREn-SC; 2003.

15. Bocchi SCM. O papel do enfermeiro como educador junto a cuidadores familiares de pessoas com AVC. Rev Bras Enferm 2004; 57(5): 569-73.

16. Martin GM, Gamble GL. Prescrição em medicina física e reabilitação. In: Kottke FI, Lehmann JF. Tratado de medicina física e reabilitação de Krusen. $4^{a}$ ed. São Paulo: Manole; 1994. p.639-47.

17. Erdmann AL, Meirelles BHS, Sousa FGM. A produção do conhecimento: o diálogo entre os diferentes saberes. Rev Bras Enfrerm 2006; 59(4): 560-4. 